\title{
Thin-shell wormholes in de Rham-Gabadadze-Tolley massive gravity
}

\author{
Takol Tangphati ${ }^{1, \mathrm{a}}$, Auttakit Chatrabhuti ${ }^{1, \mathrm{~b}}$, Daris Samart ${ }^{2, \mathrm{c}}$, Phongpichit Channuie $\mathrm{i}^{3,4,5,6, \mathrm{~d}}$ \\ ${ }^{1}$ Department of Physics, Faculty of Science, Chulalongkorn University, Bangkok 10330, Thailand \\ 2 Department of Physics, Faculty of Science, Khon Kaen University, 123 Mitraphap Road, Khon Kaen 40002, Thailand \\ ${ }^{3}$ College of Graduate Studies, Walailak University, Thasala, Nakhon Si Thammarat 80160, Thailand \\ ${ }^{4}$ School of Science, Walailak University, Thasala, Nakhon Si Thammarat 80160, Thailand \\ ${ }^{5}$ Research Group in Applied, Computational and Theoretical Science (ACTS), Walailak University, Thasala, Nakhon Si Thammarat 80160, \\ Thailand \\ ${ }^{6}$ Thailand Center of Excellence in Physics, Ministry of Higher Education, Science, Research and Innovation, Bangkok 10400, Thailand
}

Received: 31 December 2019 / Accepted: 29 July 2020 / Published online: 11 August 2020

(C) The Author(s) 2020

\begin{abstract}
In this work, we study the thin-shell wormholes in dRGT massive gravity. In order to glue two bulks of the spacetime geometry, we first derive junction conditions of the dRGT spacetime. We obtain the dynamics of the spherical thin-shell wormholes in the dRGT theory. We show that the massive graviton correction term of the dRGT theory in the Einstein equation is represented in terms of the effective anisotropic pressure fluid. However, if there is only this correction term, without invoking exotic fluids, we find that the thin-shell wormholes cannot be stabilized. We then examine the stability conditions of the wormholes by introducing four existing models of the exotic fluids at the throat. In addition, we analyze the energy conditions for the thinshell wormholes in the dRGT massive gravity by checking the null, weak, and strong conditions at the wormhole throat. We show that in general the classical energy conditions are violated by introducing all existing models of the exotic fluids. Moreover, we quantify the wormhole geometry by using the embedding diagrams to represent a thin-shell wormhole in the dRGT massive gravity.
\end{abstract}

\section{Introduction}

General theory of relativity provides an elegant mathematical description of the spacetime geometry. One of the viable solutions to Einstein's field equations yields a static and spherically symmetric black hole [1]. The recent detection

\footnotetext{
a e-mail: ta_kol@hotmail.com

be-mail: auttakit@sc.chula.ac.th

c e-mail: dsamart82@gmail.com

de-mail: channuie@gmail.com (corresponding author)
}

of gravitational waves (GWs) [2] confirmed that stellar-mass black holes really exist in Nature. Interestingly, Ludwing Flamm [3] realized in 1916 that Einstein's equations exhibit another solution currently known as a white hole. However, unlike black holes, it was believed that white holes eject matter and light from their event horizon. These two solutions could represent two different regions in spacetime connected by a conduit. This conduit was named later a "bridge" and in 1935, Einstein and Rosen used the theory of general relativity to propose the existence of "bridges" through spacetime [4]. Historically some decades later, Misner and Wheeler first introduced the term "wormhole" in Ref. [5]. Since then, wormholes become a focus on studying new realms of research. However, an original version of wormholes was later ruled out because they are not traversable. This means that its throat opens and closes so quickly.

However, in order to prevent the wormhole's throat from closing, one can add a scalar field coupled to gravity. This new ingredient provides a more general class of wormholes firstly proposed by Ellis [6] and independently by Bronnikov [7]. However, the main problem of wormholes is that they are supported by exotic matter; a kind of matter which violates the known energy conditions. Some conditions introduced by Morris and Throne in 1988 for the wormholes to be traversable can be found in Ref. [8]. These solutions are obtained by considering an unusual type of matter which can maintain the structure of the wormhole. In addition, this exotic matter with negative energy density satisfies the flareout condition and violates weak energy condition $[8,9]$. Thinshell wormholes have been studied and analyzed in several contexts involving particular models of exotic matters and theories of gravity, e.g. see Refs. [10-36] and the references therein. 
In the present work, we study the thin-shell wormholes in dRGT massive gravity. The study of massive gravity has begun prior to the discovery of the accelerated expansion of the universe. In 1939, Fierz and Pauli used a linear theory of massive gravity as a mass term of the graviton [37]. Unfortunately, there are some flaws of the proposed model [38,39] where the asymptotic-massless limits of the linear theory do not satisfy the GR prediction. Later on, the author of Ref. [40] suggested that the nonlinear approach in the massive gravity theory might be able solve the problem. However, this inevitably leads to a new problem called the Boulware-Deser (BD) ghost [41]. In 2010, the BD ghost was completely eliminated by a new nonlinear version of massive gravity proposed by de Rham, Gabadadze, and Tolley (dRGT) [42]. Since then the dRGT was targeted as one of the compelling scenarios when studying the universe on a cosmic scale. Additionally, there have been many interesting articles regarding the applications of the dRGT massive gravity to the exotic objects, e.g., black holes [43-45].

In this work, we study the thin-shell wormholes in dRGT massive gravity. The structure of the present work is as follows: we first present a short review of the dRGT model of the nonlinear massive gravity in Sect. 2. In Sect. 3, we consider the mathematical setup in order to study the thin-shell wormhole. We study junction conditions allowing one to glue two identical dRGT spacetimes. In addition, we make stability analyses of the dRGT thin-shell wormhole by considering four existing exotic fluid models in Sect. 4. We also check the null, weak, and strong conditions at the wormhole throat for all models present in Sect. 5. Moreover, we quantify the wormhole geometry by using the embedding diagrams to represent a thin-shell wormhole in the dRGT massive gravity in Sect. 6. Finally, we discuss our main results and conclude our findings in the last section. In this work, we use the geometrical unit such that $G=1$.

\section{A short recap of massive gravity}

In this section, we assume that the universe is undergoing an accelerating phase $[46,47]$. To begin the formalism, let us follow the work proposed in Ref. [42] and first define the tensor $H_{\mu \nu}$ as the covariantization of the metric perturbation, $g_{\mu \nu}=\eta_{\mu \nu}+h_{\mu \nu}=H_{\mu \nu}+\eta_{a b} \partial_{\mu} \phi^{a} \partial_{\nu} \phi^{b}$. Note that the four Stückelberg fields $\phi^{a}$ transform as scalars, and $\eta_{a b}=$ $(-1,1,1,1)$. The helicity- 0 mode $\pi$ of the graviton can be obtained by expressing $\phi^{a}=\left(x^{a}-\eta^{a \mu} \partial_{\mu} \pi\right)$ such that

$H_{\mu \nu}=h_{\mu \nu}+2 \Pi_{\mu \nu}-\eta^{\alpha \beta} \Pi_{\mu \alpha} \Pi_{\beta \nu}$,

with $\Pi_{\mu \nu}=\partial_{\mu} \partial_{\nu} \pi$. The authors of Ref. [42] define the tensor quantity $\mathcal{K}_{v}^{\mu}(g, H)$ as follows:
$\mathcal{K}_{\nu}^{\mu}(g, H)=-\sum_{n=1}^{\infty} d_{n}\left(H^{n}\right)_{\nu}^{\mu}$, with $d_{n}=\frac{(2 n) !}{(1-2 n)(n !)^{2} 4^{n}}$,

where $H_{v}^{\mu}=g^{\mu \alpha} H_{\alpha \nu}$ and $\left(H^{n}\right)_{\nu}^{\mu}=H_{\alpha_{1}}^{\mu} H_{\alpha_{2}}^{\alpha_{1}} H_{\alpha_{3}}^{\alpha_{2}} \ldots H_{v}^{\alpha_{n-1}}$ denotes the product of $n$ tensors $H_{\beta}^{\alpha}$. Therefore, the action representing the dRGT model on the manifold $\mathcal{M}$ is given by $^{1}$

$S=\int_{M} \mathrm{~d}^{4} x \sqrt{-g} \frac{1}{16 \pi G}\left(R+m_{g}^{2} \mathcal{U}\left(g, \phi^{a}\right)\right)$,

where $\sqrt{-g}$ is the volume element in 4-dimensional manifold $\mathcal{M}$ and the potential $\mathcal{U}$ is defined by

$\mathcal{U}=\mathcal{U}_{2}+\alpha_{3} \mathcal{U}_{3}+\alpha_{4} \mathcal{U}_{4}$

where $\mathcal{U}_{2}, \mathcal{U}_{3}$ and $\mathcal{U}_{4}$ are given by

$\mathcal{U}_{2}=[\mathcal{K}]^{2}-\left[\mathcal{K}^{2}\right]$,

$\mathcal{U}_{3}=[\mathcal{K}]^{3}-3[\mathcal{K}]\left[\mathcal{K}^{2}\right]+2\left[\mathcal{K}^{3}\right]$,

$\mathcal{U}_{4}=[\mathcal{K}]^{4}-6[\mathcal{K}]^{2}\left[\mathcal{K}^{2}\right]+8[\mathcal{K}]\left[\mathcal{K}^{3}\right]+3\left[\mathcal{K}^{2}\right]^{2}-6\left[\mathcal{K}^{4}\right]$.

Here a bracket [ ] represents the trace of the tensor, $[\mathcal{K}] \equiv$ $\mathcal{K}_{\mu}^{\mu},\left[\mathcal{K}^{2}\right] \equiv \mathcal{K}_{\alpha_{1}}^{\mu} \mathcal{K}_{\mu}^{\alpha_{1}},\left[\mathcal{K}^{3}\right] \equiv \mathcal{K}_{\alpha_{1}}^{\mu} \mathcal{K}_{\beta_{1}}^{\alpha_{1}} \mathcal{K}_{\mu}^{\beta_{1}}$ and $\left[\mathcal{K}^{4}\right] \equiv$ $\mathcal{K}_{\alpha_{1}}^{\mu} \mathcal{K}_{\beta_{1}}^{\alpha_{1}} \mathcal{K}_{\alpha_{2}}^{\beta_{1}} \mathcal{K}_{\mu}^{\alpha_{2}}$. Note here that parameters $\alpha_{3}$ and $\alpha_{4}$ of the dRGT theory can be related to the graviton mass. Performing variation of the gravitational action in Eq. (3) with respect to the metric, $g_{\mu \nu}$, yields the Einstein equation of the dRGT massive gravity,

$G_{\mu \nu}+m_{g}^{2} X_{\mu \nu}=0$,

where $X_{\mu \nu}$ is defined by

$$
\begin{aligned}
X_{\mu \nu}= & \frac{1}{\sqrt{-g}} \frac{\delta \sqrt{-g} \mathcal{U}}{\delta g^{\mu \nu}} \\
= & \mathcal{K}_{\mu \nu}-\alpha\left[\left(\mathcal{K}^{2}\right)_{\mu \nu}-[\mathcal{K}] \mathcal{K}_{\mu \nu}+\frac{1}{2} g_{\mu \nu}\left([\mathcal{K}]^{2}-\left[\mathcal{K}^{2}\right]\right)\right] \\
& +3 \beta\left[\left(\mathcal{K}^{3}\right)_{\mu \nu}-[\mathcal{K}]\left(\mathcal{K}^{2}\right)_{\mu \nu}+\frac{1}{2} \mathcal{K}_{\mu \nu}\left([\mathcal{K}]^{2}-\left[\mathcal{K}^{2}\right]\right)\right. \\
& \left.-\frac{1}{6} g_{\mu \nu}\left([\mathcal{K}]^{3}-3[\mathcal{K}]\left[\mathcal{K}^{2}\right]+2\left[\mathcal{K}^{3}\right]\right)\right]
\end{aligned}
$$

where the parameters $\alpha$ and $\beta$ are related to $\alpha_{3,4}$ from the action in Eq. (3) via

$\alpha=1+3 \alpha_{3}, \quad \beta=\alpha_{3}+4 \alpha_{4}$.

In addition, one finds that the Einstein equation of the dRGT massive gravity is reduced to the standard GR by setting $m_{g} \rightarrow 0$. Having used the static and spherically symmetric

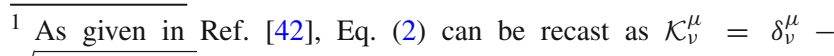
$\sqrt{\partial_{\mu} \phi^{a} \partial_{\nu} \phi^{b} \eta_{a b}}$, yielding a square root structure in the full Lagrangian. 
spacetime, we obtain an explicit form of the line element of the dRGT massive gravity which reads $[42,48]$

$\mathrm{d} s^{2}=g_{\mu \nu} \mathrm{d} x^{\mu} \mathrm{d} x^{\nu}=-f(r) \mathrm{d} t^{2}+\frac{\mathrm{d} r^{2}}{f(r)}+r^{2} \mathrm{~d} \Omega^{2}$,

where $\mathrm{d} \Omega^{2}=\mathrm{d} \theta^{2}+\sin ^{2} \theta \mathrm{d} \phi^{2}$ and the function $f(r)$ is the vacuum solution without any kind of matter. The latter can be written as

$f(r)=1-\frac{2 M}{r}-\frac{\Lambda r^{2}}{3}+\gamma r+\zeta$,

where $M$ is the mass parameter, $\Lambda$ is the effective cosmological constant, and $\gamma$ and $\zeta$ are new parameters and they are linear combinations of the parameters in the dRGT massive gravity via the following relations:

$$
\begin{aligned}
& \Lambda \equiv-3 m_{g}^{2}(1+\alpha+\beta), \quad \gamma \equiv-m_{g}^{2} k(1+2 \alpha+3 \beta), \\
& \zeta \equiv m_{g}^{2} k^{2}(\alpha+3 \beta)
\end{aligned}
$$

The behaviors of how a function $f(r)$ depends on $r$ is displayed in Fig. 1. According to the Einstein equation of the dRGT massive gravity in Eq. (6), one might identify the $m_{g}^{2} X_{\mu \nu}$ term as the effective energy-momentum tensor. Using an explicit form of the metric tensors in Eq. (10), allows us to directly compute components of $m_{g}^{2} X_{\mu \nu}$ in Eq. (7). The components of $m_{g}^{2} X_{v}^{\mu}$ read [49-51]

$$
\begin{aligned}
\rho_{g}(r) & \equiv-\frac{m_{g}^{2}}{8 \pi G} X_{t}^{t} \\
& =\frac{m_{g}^{2}}{8 \pi G}\left(\frac{3 r-2 k}{r}+\frac{\alpha(3 r-k)(r-k)}{r^{2}}+\frac{3 \beta(r-k)^{2}}{r^{2}}\right), \\
p_{g}^{(r)}(r) & \equiv-\frac{m_{g}^{2}}{8 \pi G} X_{r}^{r} \\
& =\frac{m_{g}^{2}}{8 \pi G}\left(\frac{3 r-2 k}{r}+\frac{\alpha(3 r-k)(r-k)}{r^{2}}+\frac{3 \beta(r-k)^{2}}{r^{2}}\right), \\
p_{g}^{(\theta, \phi)}(r) & \equiv-\frac{m_{g}^{2}}{8 \pi G} X_{\theta, \phi}^{\theta, \phi} \\
& =\frac{m_{g}^{2}}{8 \pi G}\left(\frac{3 r-k}{r}+\frac{\alpha(3 r-2 k)}{r}+\frac{3 \beta(r-k)}{r}\right) .
\end{aligned}
$$

With the help of Eq. (11), the parameters $k, \alpha$ and $\beta$ are rewritten in terms of $\Lambda, \gamma$ and $\zeta$ by

$$
\begin{aligned}
k & =\frac{\gamma+\sqrt{\gamma^{2}+\left(m_{g}^{2}+\Lambda\right) \zeta}}{m_{g}^{2}+\Lambda}, \\
\alpha & =-\frac{\gamma^{2}+\left(2 m_{g}^{2}+\Lambda\right) \zeta-\gamma \sqrt{\gamma^{2}+\left(m_{g}^{2}+\Lambda\right) \zeta}}{m_{g}^{2} \zeta}, \\
\beta & =\frac{2 \Lambda}{3 m_{g}^{2}}+\frac{\gamma^{2}+m_{g}^{2} \zeta-\gamma \sqrt{\gamma^{2}+\left(m_{g}^{2}+\Lambda\right) \zeta}}{m_{g}^{2} \zeta} .
\end{aligned}
$$

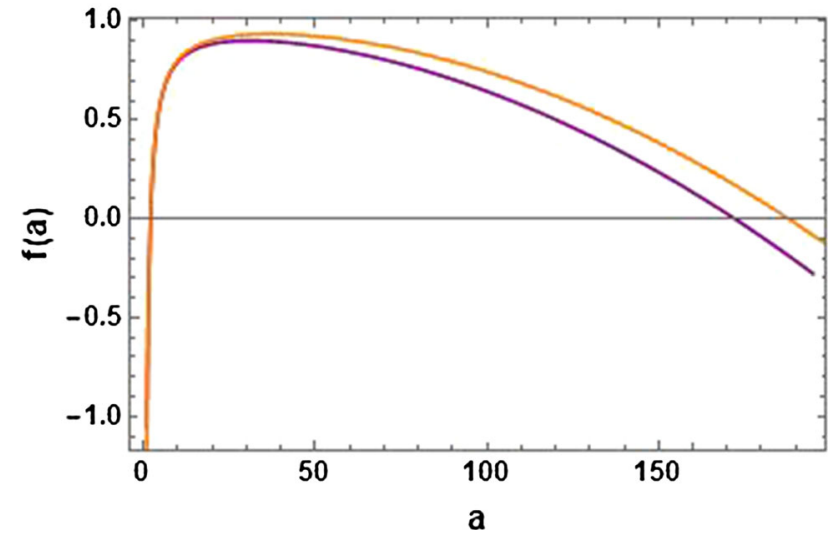

Fig. 1 Behaviors of $f(r)$ against $r$. For simplicity, we have used $M=1.0, G=1.0, \zeta=0.0$. We labeled lines with orange and purple colors for $\Lambda=0.0001, \gamma=0.001$ and $\Lambda=0.0001, \gamma=0.00002$, respectively

In addition, we found that the parameters $k, \alpha$ and $\beta$ are finite in the $\zeta \rightarrow 0$ limit, i.e.,

$k=\frac{2 \gamma}{m_{g}^{2}+\Lambda}, \quad \alpha=-3 \beta=-\frac{3}{2}-\frac{\Lambda}{2 m_{g}^{2}}$.

In this limit, the above relations are well defined for tuning the positive value and the smallness of the effective cosmological constant, $\Lambda$, constrained by the observed values from the cosmological data. More importantly, it has been shown that the effective energy-momentum tensor, $X_{\mu \nu}$, exhibits a behavior like anisotropic dark energy fluid i.e., $p_{g}^{(r)}=-\rho_{g}$; see more detailed discussions and applications in Refs. [4951].

We close this section by adding matter fluid to the Einstein equation. By using the standard method in GR, the Einstein equation with the (fluid) matter field of the dRGT massive gravity is given by

$$
\begin{aligned}
G_{\mu \nu} & =8 \pi G T_{\mu \nu}, \quad T_{\mu \nu}=T_{\mu \nu}^{(f)}+T_{\mu \nu}^{(g)}, \\
T_{\mu \nu}^{(f)} & =(\sigma+p) u_{\mu} u_{\nu}+p g_{\mu \nu}, \\
T_{\mu \nu}^{(g)} & =-\frac{m_{g}^{2}}{8 \pi G} X_{\mu \nu} \\
& =\left(\rho_{g}+p_{g}^{(\perp)}\right) u_{\mu} u_{\nu}+p_{g}^{(\perp)} g_{\mu \nu}+\left(p_{g}^{(r)}-p_{g}^{(\perp)}\right) \chi_{\mu} \chi_{\nu},
\end{aligned}
$$

where $\sigma$ and $p$ are, respectively, the energy density and the isotropic pressure of the fluid matter, while the components of the effective energy-momentum tensor of the dRGT massive gravity $\left(T_{\mu \nu}^{(g)}\right)$ are already given in Eqs. (12-14). The $u_{\mu}$ is a time-like unit vector, the $\chi_{\mu}$ is the space-like unit vector orthogonal to the $u_{\mu}$ and the angular plane and $p_{g}^{(\perp)}=p_{g}^{(\theta)}=p_{g}^{(\phi)}$. The total energy-momentum tensor can be recast in the following form:

$T_{v}^{\mu}=T_{v}^{(f), \mu}+T_{v}^{(g), \mu}$ 


$$
=\left(\begin{array}{cccc}
-\sigma-\rho_{g} & 0 & 0 & 0 \\
0 & p+p_{g}^{(r)} & 0 & 0 \\
0 & 0 & p+p_{g}^{(\perp)} & 0 \\
0 & 0 & 0 & p+p_{g}^{(\perp)}
\end{array}\right) .
$$

Moreover, the energy-momentum tensor given in Eq. (17) satisfy the conservation law:

$\nabla^{\mu} T_{\mu \nu}=0, \quad \nabla^{\mu} T_{\mu \nu}^{(f)}=0, \quad \nabla^{\mu} T_{\mu \nu}^{(g)}=0$.

\section{The thin-shell wormholes in dRGT spacetimes}

\subsection{Junction conditions in dRGT theory}

In this work, the wormhole helps joining two different dRGT spacetimes. It behaves like a surface between two bulks and is called thin shell $[52,53]$. In order to join two different manifolds, we will construct a thin-shell wormhole of the dRGT massive gravity following the standard approach proposed in Refs. [54-56]. Consider two distinct spacetime manifolds, $\mathcal{M}_{+}$and $\mathcal{M}_{-}$, with metrics given by $g_{\mu \nu}^{+}\left(x_{+}^{\mu}\right)$ and $g_{\mu \nu}^{-}\left(x_{-}^{\mu}\right)$, in terms of independently defined coordinate systems $x_{+}^{\mu}$ and $x_{-}^{\mu}$. The manifolds are bounded by hypersurfaces $\Sigma_{+}$and $\Sigma_{-}$with induced metrics $h_{a b}^{+}$and $h_{a b}^{-}$, respectively. Regarding the Darmois-Israel formalism, the coordinates on $\mathcal{M}$ can be chosen as $x^{\mu}=(t, r, \theta, \phi)$, while for the coordinates on the induced metric $\Sigma$, we write $y^{a}=(\tau, \theta, \phi)$, these being the intrinsic coordinates. Note that the hypersurfaces are isometric, i.e., $h_{a b}^{+}\left(y^{a}\right)=h_{a b}^{-}\left(y^{a}\right)=h_{a b}\left(y^{a}\right)$. A single manifold $\mathcal{M}$ is obtained by gluing together $\mathcal{M}_{+}$and $\mathcal{M}_{-}$ at their boundaries, i.e., $\mathcal{M}=\mathcal{M}_{+} \cup \mathcal{M}_{-}$, with the natural identification of the boundaries $\Sigma_{+}=\Sigma_{-}=\Sigma$.

We describe two different manifolds as follows $[55,56]$ :

$\mathcal{M}_{ \pm}=\left\{x_{ \pm}^{\mu} \mid t_{ \pm} \geq T_{ \pm}(\tau)\right.$ and $\left.r \geq a(\tau)\right\}$,

where the plus (minus) sign means the upper (the lower) spacetime manifold. Before considering the junction condition for matching the boundaries between two different manifolds, it is worth mentioning that at the end of Sect. 2, we have written the Einstein equations including matter terms for the whole theory, in the bulk spacetime. However, in the latter, the matter is assumed to exist only on the thin shell comprising the throat. The line elements of the manifolds are given by

$\mathrm{d} s_{ \pm}^{2}=g_{\mu \nu}^{ \pm} \mathrm{d} x^{\mu} \mathrm{d} x^{\nu}=-f_{ \pm}(r) \mathrm{d} t^{2}+\frac{\mathrm{d} r^{2}}{f_{ \pm}(r)}+r^{2} \mathrm{~d} \Omega^{2}$.

Both different manifolds are linked by the (co-moving) thin shell and the hypersurface $\Sigma$ is parametrized by [55-58]

$\Sigma=\left\{y^{a} \mid t_{ \pm}=T_{ \pm}(\tau)\right.$ and $\left.r=a(\tau)\right\}$.
Thus the line element of the thin shell reads

$$
\begin{aligned}
\mathrm{d} s_{\Sigma}^{2} & =g_{\alpha \beta} \mathrm{d} x^{\alpha} \mathrm{d} x^{\beta}=g_{\alpha \beta}\left(\frac{\partial x^{\alpha}}{\partial y^{a}} \mathrm{~d} y^{a}\right)\left(\frac{\partial x^{\beta}}{\partial y^{b}} \mathrm{~d} y^{b}\right) \\
& =h_{a b} \mathrm{~d} y^{a} \mathrm{~d} y^{b}=-\mathrm{d} \tau^{2}+a^{2}(\tau) \mathrm{d} \Omega^{2},
\end{aligned}
$$

where $y^{a}=y^{a}\left(x^{\mu}\right)$ is a coordinate on the hypersurface $\Sigma$ and $h_{a b}$ is called the induced metric or the first fundamental form on the hypersurface $\Sigma$, [55-57]

$h_{a b} \equiv g_{\alpha \beta} e_{a}^{\alpha} e_{b}^{\beta}$.

It is worth noting here that the throat must satisfy by the Israel junction conditions which provide the following coordinate choice:

$-f_{ \pm}(a) \dot{T}_{ \pm}^{2}+\frac{\dot{a}^{2}}{f_{ \pm}(a)}=1, \quad \forall \tau$,

where dots denote derivative with respect to $\tau(\mathrm{d} / \mathrm{d} \tau)$ and $\tau$ simply is a local time on the thin shell. The induced metric is a tangent component of $g_{\alpha \beta}$ on the hypersurface $\Sigma$. Then the normal vector component $n_{\alpha}$ of the metric tensor on the hypersurface is defined as follows [55-57]:

$n_{\alpha} \equiv \frac{F(r, a(\tau))_{, \alpha}}{\left|F(r, a(\tau))_{, \beta} F(r, a(\tau))^{, \beta}\right|^{1 / 2}}$,

where $F(r, a(\tau)) \equiv r-a(\tau)=0$ is the hypersurface function and $a(\tau)$ is the throat radius of the thin-shell wormhole. Note that the Greek indices $\left(x^{\alpha}, x^{\beta}, \ldots\right)$ are the coordinates on the manifold $M_{ \pm}$, while the Latin indices $\left(x^{a}, x^{b}, \ldots\right)$ are the coordinates on the hypersurface $\Sigma$. The metric tensor on the hypersurface can be split into two parts [55-57] as

$g_{a b}=h_{a b}+\epsilon n_{a} n_{b}$,

where $\epsilon$ represents the types of thin shell with $\epsilon=-1,0,+1$ being the space-like, null-like and time-like, respectively.

Next we will briefly derive the junction conditions on the hypersurface between the boundaries of two different manifolds $(\partial \mathcal{M})$. It is well known that in order to connect two manifolds one needs to add the action of the boundary terms or the Gibbons-Hawkings terms into the total action. Then the total gravitational action of the dRGT massive gravity with the matter fields and the boundary terms is given by [54,59]

$$
\begin{aligned}
S_{\text {total }}= & \int_{\mathcal{M}_{+}} \mathrm{d}^{4} x \sqrt{-g^{+}} \\
& \left(\frac{1}{16 \pi G}\left(R^{+}+m_{g}^{2} \mathcal{U}\left(g^{+}, \phi^{a}\right)\right)+\mathcal{L}_{\text {matter }}^{+}\right) \\
& +\frac{1}{8 \pi G} \int_{\partial \mathcal{M}_{+}} \mathrm{d}^{3} y \sqrt{-h^{+}} K^{+} \\
& +\int_{\mathcal{M}_{-}} \mathrm{d}^{4} x \sqrt{-g^{-}} \\
& \left(\frac{1}{16 \pi G}\left(R^{-}+m_{g}^{2} \mathcal{U}\left(g^{-}, \phi^{a}\right)\right)+\mathcal{L}_{\text {matter }}^{-}\right)
\end{aligned}
$$




$$
\begin{aligned}
& +\frac{1}{8 \pi G} \int_{\partial \mathcal{M}_{-}} \mathrm{d}^{3} y \sqrt{-h^{-}} K^{-} \\
& +\int_{\Sigma} \mathrm{d}^{3} y \sqrt{-h} \mathcal{L}_{\text {matter }}^{\Sigma}
\end{aligned}
$$

where $\sqrt{-h}$ is the volume element on the 3-dimensional hypersurface and $K$ is the trace of the extrinsic curvature $K_{a b}$ on the thin shell with $K \equiv K_{a}^{a}=h_{a b} K_{a}^{b}$. Here $\mathcal{L}_{\text {matter }}^{\Sigma}=\mathcal{L}_{f}^{\Sigma}+\mathcal{L}_{g}^{\Sigma}$ contains two types of fluids (perfect fluid, $\mathcal{L}_{f}^{\Sigma}$ and massive gravity fluid, $\mathcal{L}_{f}^{\Sigma}$ ) which are localized on the hypersurface. The extrinsic curvature can be calculated via the following equation:

$K_{a b}=-n_{\alpha}\left[\frac{\mathrm{d}^{2} x^{\alpha}}{\mathrm{d} y^{a} \mathrm{~d} y^{b}}+\Gamma_{\beta \gamma}^{\alpha} \frac{\mathrm{d} x^{\beta}}{\mathrm{d} y^{a}} \frac{\mathrm{d} x^{\gamma}}{\mathrm{d} y^{b}}\right]$.

Let us first discuss the matter Lagrangian on the hypersurface,

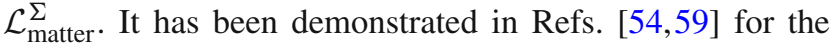
scalar field matter case that the Lagrangian of the matter field on the hypersurface has the same form of that in the bulk, but the metric tensor $g_{\mu \nu}$ in the bulk is replaced by the induced metric on the hypersurface, $h_{a b}$.

Varying the total gravitational action in Eq. (29) with respect to the induced metric tensor, $h^{a b}$ and using the standard technique of the hypersurface in GR, the junction condition is given by

$\delta_{b}^{a} \Delta K-\Delta K_{b}^{a}=8 \pi G S_{b}^{a}$,

where we have the notation $\Delta A \equiv A_{+}-A_{-}$. The new effective energy-momentum tensor $S_{a b}$ on the thin shell is defined by

$S_{b}^{a} \equiv t_{b}^{a}+Y_{b}^{a}$

In addition, the definition of the energy-momentum tensor of the fluid on the thin shell, $t_{b}^{a}$ takes the form

$t_{b}^{a}=-\frac{1}{\sqrt{-h}} \frac{\delta}{\delta h_{a}^{b}}\left(\sqrt{-h} \mathcal{L}_{f}^{\Sigma}\right)=(\sigma+p) u^{a} u_{b}+p h_{b}^{a}$.

For the massive gravity fluid, the $Y_{b}^{a}$ tensor is given by

$$
\begin{aligned}
Y_{b}^{a} & =-\frac{1}{\sqrt{-h}} \frac{\delta}{\delta h_{a}^{b}}\left(\sqrt{-h} \mathcal{L}_{g}^{\Sigma}\right) \\
& =\left(\rho_{g}+p_{g}^{(\perp)}\right) u^{a} u_{b}+p_{g}^{(\perp)} h_{b}^{a} .
\end{aligned}
$$

Furthermore, it is very convenient to represent the $S_{b}^{a}$ tensor in the matrix form,

$$
\begin{aligned}
S_{b}^{a} & \equiv\left(\begin{array}{ccc}
-\rho_{\mathrm{eff}} & 0 & 0 \\
0 & P_{\mathrm{eff}} . & 0 \\
0 & 0 & P_{\text {eff. }}
\end{array}\right) \\
& =\left(\begin{array}{ccc}
-\sigma-\rho_{g} & 0 & 0 \\
0 & p+p_{g}^{(\perp)} & 0 \\
0 & 0 & p+p_{g}^{(\perp)}
\end{array}\right),
\end{aligned}
$$

where the explicit forms of the $\rho_{g}$ and $p_{g}^{(\perp)}=p_{g}^{(\theta, \phi)}$ are given in Eqs. (12) and (14). We will see in the latter case that the equation of motion of the dRGT massive gravity wormholes takes a very simple form, like the standard GR case with two types of fluids.

3.2 The thin-shell wormhole dynamics in dRGT spacetimes

We next consider the relation between the thin-shell wormhole on the hypersurface $\Sigma$ and the spacetime on manifold $\mathcal{M}_{ \pm}$. Considering the parameterization of the coordinate on the hypersurface in Eq. (23), we find

$-\mathrm{d} \tau^{2}=-f_{ \pm}(a) d T_{ \pm}^{2}+\frac{\mathrm{d} a^{2}}{f_{ \pm}(a)}$

and

$a^{2} \mathrm{~d} \Omega^{2}=r^{2} \mathrm{~d} \Omega^{2}$

The relation between $T$ and $\tau$ is given by

$\dot{T}_{ \pm}=\frac{1}{f_{ \pm}(a)} \sqrt{f_{ \pm}(a)+\dot{a}^{2}}$

and

$\ddot{T}_{ \pm}=-\frac{\dot{f}_{ \pm}}{f_{ \pm}^{2}} \sqrt{f_{ \pm}+\dot{a}^{2}}+\frac{2 \dot{a} \ddot{a}+\dot{f}_{ \pm}}{2 f_{ \pm} \sqrt{f_{ \pm}+\dot{a}^{2}}}$,

where $\dot{f}=\frac{\mathrm{d} f}{\mathrm{~d} \tau}=\frac{\mathrm{d} f(a)}{\mathrm{d} a} \frac{\mathrm{d} a}{\mathrm{~d} \tau}=f^{\prime}(a) \dot{a}$ and a prime denotes the first derivative with respect to $a$. Now we are ready to compute the non-vanishing components of the extrinsic curvature of the wormhole in dRGT massive gravity. Having used the line element in Eq. (24), one obtains the non-vanishing components of $K_{b}^{a}$ as

$K_{\tau}^{\tau \pm}= \pm \frac{1}{\sqrt{f_{ \pm}+\dot{a}^{2}}}\left(\ddot{a}+\frac{f_{ \pm}^{\prime}}{2}\right)$,

$K_{\theta}^{\theta \pm}=K_{\phi}^{\phi \pm}= \pm \frac{1}{a}\left(\sqrt{f_{ \pm}+\dot{a}^{2}}\right)$.

We note that the extrinsic curvature is a diagonal matrix. It is worth mentioning the parameters in the dRGT massive gravity. By using the observational constraints on the dRGT theory, we find 
$\zeta=0, \quad \Rightarrow \quad \alpha=-3 \beta=-\frac{3}{2}-\frac{\Lambda}{2 m_{g}^{2}}, \quad k=\frac{2 \gamma}{m_{g}^{2}+\Lambda}$.

Using Eqs. (12) and (14) with $r=a$ at the boundaries $\partial \mathcal{M}_{ \pm}$, the components of the effective energy-momentum tensor, $S_{b}^{a}$, in Eq. (35) are given by the following explicit expressions:

$$
\begin{aligned}
S_{\tau}^{\tau} & =-\rho_{\text {eff. }}=-\sigma-\rho_{g}(a) \\
& =-\sigma+\frac{1}{8 \pi G}\left(\frac{2 \gamma}{a}-\Lambda\right), \\
S_{\theta}^{\theta} & =S_{\phi}^{\phi}=P_{\text {eff. }}=p+p_{g}^{(\perp)}(a) \\
& =p+\frac{1}{8 \pi G}\left(\frac{\gamma}{a}-\Lambda\right) .
\end{aligned}
$$

Setting $\zeta=0$, we find that there is only one free parameter of the dRGT massive gravity since the graviton mass, $m_{g}$ and the cosmological constant, $\Lambda$ can be fixed by using the observed values of those two quantities.

In this work, we assume the $Z_{2}$ symmetry between two bulk spacetime manifolds. This means that $f_{+}(a)=$ $f_{-}(a)=f(a), \Lambda_{+}=\Lambda_{-}=\Lambda$, and $\gamma_{+}=\gamma_{-}=\gamma$. The ( $\tau \tau)$ component of the junction condition of the thin-shell wormhole in Eq. (31) reads

$\frac{2}{a}\left(\sqrt{f+\dot{a}^{2}}\right)=-8 \pi G \sigma+\left(\frac{2 \gamma}{a}-\Lambda\right)$.

On the other hand, the angular component of Eq. (31) is given by

$\frac{1}{\sqrt{f+\dot{a}^{2}}}\left(2 \ddot{a}+f^{\prime}\right)=8 \pi G p+\left(\frac{\gamma}{a}-\Lambda\right)$.

In addition, the continuity of the perfect fluid matter gives a relation between the energy density and pressure on the thin shell as

$\frac{\mathrm{d}}{\mathrm{d} \tau}(a \sigma)+p \frac{\mathrm{d} a}{\mathrm{~d} \tau}=0$

It is also written in terms of the first order derivative of $\sigma$ with respect to $a$ as

$\frac{\mathrm{d} \sigma}{\mathrm{d} a}=-\left(\frac{\sigma+p}{a}\right)$.

The second order derivative of $\sigma$ with respect to $a$ yields

$\frac{\mathrm{d}^{2} \sigma}{\mathrm{d} a^{2}}=\frac{\sigma+p}{a^{2}}\left(2+\frac{\mathrm{d} p}{\mathrm{~d} \rho}\right)$,

where $p=p(\sigma)$. The above equations are useful for analyzing the stability of the wormhole with several types of the perfect fluid matters. We study their effects on particular models in the next section.

\section{Stability analysis of the dRGT thin-shell wormhole}

The stability of the wormhole can be quantified via the study of the effective potential of the wormhole dynamics. The equation of motion for determining the stability of the throat $a(\tau)$ is directly derived from Eq. (44) to obtain

$\frac{1}{2} \dot{a}^{2}+V(a)=0$

where the effective potential $V(a)$ is written by

$V(a)=\frac{1}{2} f(a)-\frac{a^{2}}{8}\left[8 \pi G \sigma-\left(\frac{2 \gamma}{a}-\Lambda\right)\right]^{2}$.

This single dynamical equation (49) completely determines the motion of the wormhole throat. Notice that if we consider only the massive gravity correction term, $m_{g}^{2} \mathcal{U}$, without invoking exotic fluids, i.e., $\sigma=0=p$, the potential (50) becomes

$V(a)=\frac{1}{2} f(a)-\frac{a^{2}}{8}\left[\frac{2 \gamma}{a}-\Lambda\right]^{2}$.

In this situation, we find that $V^{\prime \prime}\left(a_{0}\right)<0$ meaning that it is not possible to obtain stable wormholes if $\Lambda>0$. We assume that the throat of the thin-shell wormhole is static at $a=a_{0}$ and satisfies the relation

$f\left(a_{0}\right)>0$,

in order to avoid the event horizon $r_{\mathrm{EH}}$ from the wormhole, $f\left(r_{\mathrm{EH}}\right)=0$. In order to analyze the stability of the throat, we consider a small perturbation to the potential and are able to determine whether the throat is stable or not. Here the usual Taylor series expansion is applied to the potential $V(a)$ around the static radius $a_{0}$ as follows:

$$
\begin{aligned}
V(a)= & V\left(a_{0}\right)+V^{\prime}\left(a_{0}\right)\left(a-a_{0}\right)+\frac{1}{2} V^{\prime \prime}\left(a_{0}\right)\left(a-a_{0}\right)^{2} \\
& +\mathcal{O}\left(\left(a-a_{0}\right)^{3}\right) .
\end{aligned}
$$

When evaluating at the static solution $a=a_{0}$, we obtain the expected result $V\left(a_{0}\right)=0$ and $V^{\prime}\left(a_{0}\right)=0$ if $a_{0}$ is the static radius. Then Eq. (53) reduces to

$V(a)=\frac{1}{2} V^{\prime \prime}\left(a_{0}\right)\left(a-a_{0}\right)^{2}+\mathcal{O}\left(\left(a-a_{0}\right)^{3}\right)$.

Therefore, the equation of motion for the wormhole throat approximately takes the form

$\dot{a}^{2}+\frac{1}{2} V^{\prime \prime}\left(a_{0}\right)\left(a-a_{0}\right)^{2}=0$.

Using Eq. (50) with the help of Eq. (47) and Eq. (48), we find

$$
\begin{aligned}
V^{\prime \prime}\left(a_{0}\right)= & \frac{1}{2} f^{\prime \prime}\left(a_{0}\right)+\frac{\mathrm{d} p}{\mathrm{~d} \sigma}(-2 G(p+\sigma) \pi \Lambda \\
& \left.-16 G^{2} \pi^{2} \sigma(p+\sigma)+\frac{4 G \pi \gamma(p+\sigma)}{a}\right)
\end{aligned}
$$




$$
-16 G^{2} p^{2} \pi^{2}+4 G p \pi \Lambda-\frac{1}{4} \Lambda^{2} .
$$

Thus, the wormhole is stable if and only if $V^{\prime \prime}\left(a_{0}\right)>0$ where the motion of the throat is oscillatory with angular frequency $\omega=\sqrt{V^{\prime \prime}\left(a_{0}\right) / 2}$. Note that $V\left(a_{0}\right)$ has a local minimum at $a_{0}$. To carry out the analysis, we can quantify the conditions to obtain stable wormholes. In our case, we find that these parameters need to satisfy

$$
\begin{aligned}
0< & \frac{1}{2} f^{\prime \prime}\left(a_{0}\right)+\frac{\mathrm{d} p}{\mathrm{~d} \sigma}(-2 G(p+\sigma) \pi \Lambda \\
& \left.-16 G^{2} \pi^{2} \sigma(p+\sigma)+\frac{4 G \pi \gamma(p+\sigma)}{a_{0}}\right) \\
& -16 G^{2} p^{2} \pi^{2}+4 G p \pi \Lambda-\frac{1}{4} \Lambda^{2} .
\end{aligned}
$$

Next we assume four fluid models for studying the stability of the dRGT wormhole: (1) the linear model, (2) the Chaplygin gas model, (3) the generalized Chaplygin gas model and (4) the logarithm model. It has been shown in the previous section that we have only one free parameter $(\gamma)$ in the dRGT theory by using $\zeta=0$. The parameters in this work in natural units are given by

$$
\begin{aligned}
G & =6.72 \times 10^{-57} \mathrm{eV}^{-2}, \quad m_{g}=1.22 \times 10^{-22} \mathrm{eV}, \\
\Lambda & =4.33 \times 10^{-66} \mathrm{eV}^{2},
\end{aligned}
$$

where the gravitational constant $G$, the cosmological constant, $\Lambda$ are taken from review of particle physics [60] and the graviton mass is the upper bound values from the LIGOVIRGO gravitational wave observations [2]. Moreover, the free parameter, $\gamma$ of the dRGT massive gravity has been fixed by fitting rotational curves of the galaxies in several data [51] and we will use this value for the following study. The $\gamma$ parameter and the thin-shell wormhole mass read

$\gamma=6.05 \times 10^{-34} \mathrm{eV}, \quad M=3.36 \times 10^{66} \mathrm{eV}$,

where we have assumed that $M$ is roughly equal to the lower bound mass of black hole which is three times of the solar mass. Using the above numerical values, we can estimate the event horizon and the cosmological horizon via Eq. (10) by setting $f(r)=0$.

However, it is much more convenient to use the dimensionless values of the physical parameters given below:

$\Lambda=0.0001, \quad \gamma=0.001, \quad M=1$.

Note that one can easily change the units from this dimensionless parameters to the others, e.g. SI units or natural units, and vice versa. Now we consider the energy density given in Eq. (44) and write for the static case at the throat

$\sigma=\frac{1}{8 \pi G}\left(\frac{2 \gamma}{a}-\Lambda\right)-\frac{2}{8 \pi G a} \sqrt{f}$.

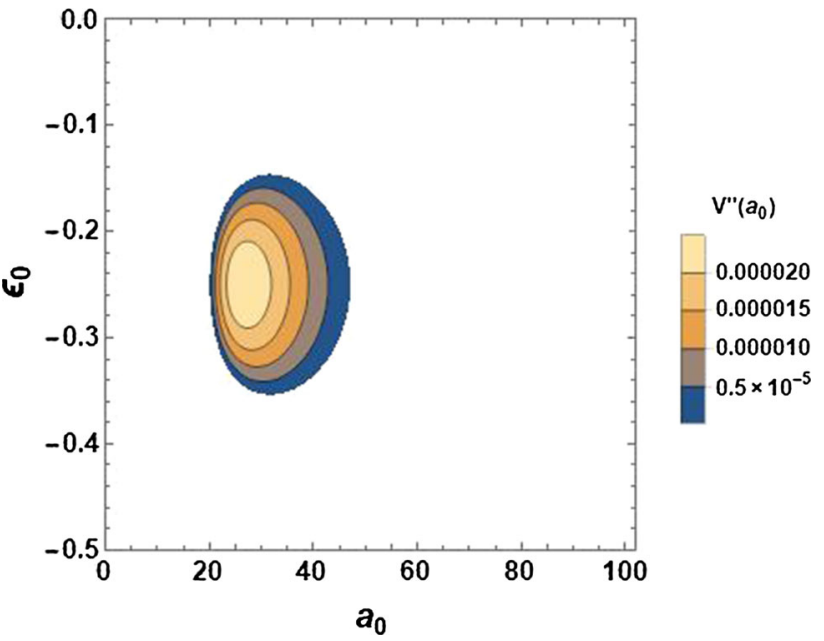

Fig. 2 The stable region of the linear model $p(\sigma)=\epsilon_{0} \sigma$. The contour shows that the constant $\epsilon_{0}$ has negative values in the throat

Here we can solve the above equation to write $\sigma$ in terms of $a$ when substituting a function $f(a)$. It was found that the stability of transparent spherically symmetric thin shells to linearized spherically symmetric perturbations about static equilibrium has been examined Ref. [61].

\subsection{Linear model}

We begin our stability analyses by considering the pressure which is proportional the energy density [56]:

$p(\sigma)=\epsilon_{0} \sigma$.

It is easy to show that

$\frac{\mathrm{d} p}{\mathrm{~d} \sigma}=\epsilon_{0}$.

Notice that the change in the pressure on the energy density is a constant. Moreover, the throat of the wormhole basically locates between the event horizon and the cosmological horizon. After substituting the above results into the stability condition (57), we find

$$
\begin{aligned}
0< & \frac{1}{2} f^{\prime \prime}\left(a_{0}\right)-\frac{1}{4}\left(\Lambda^{2}+8 \pi\left(\epsilon_{0}-1\right) \epsilon_{0} \Lambda \sigma+64 \pi^{2} \epsilon_{0}\left(1+2 \epsilon_{0}\right) \sigma^{2}\right) \\
& +\frac{4 \pi \gamma \epsilon_{0}\left(\epsilon_{0}+1\right)}{a} .
\end{aligned}
$$

In order to visualize the stability region of the model, we plot the stability contour in terms of $\epsilon_{0}$ and $a_{0}$. Our result is illustrated in Fig. 2 for the linear model. We notice that in order to satisfy the stability condition (57) the constant $\epsilon_{0}$ has negative values in the throat radius $a_{0}$. 


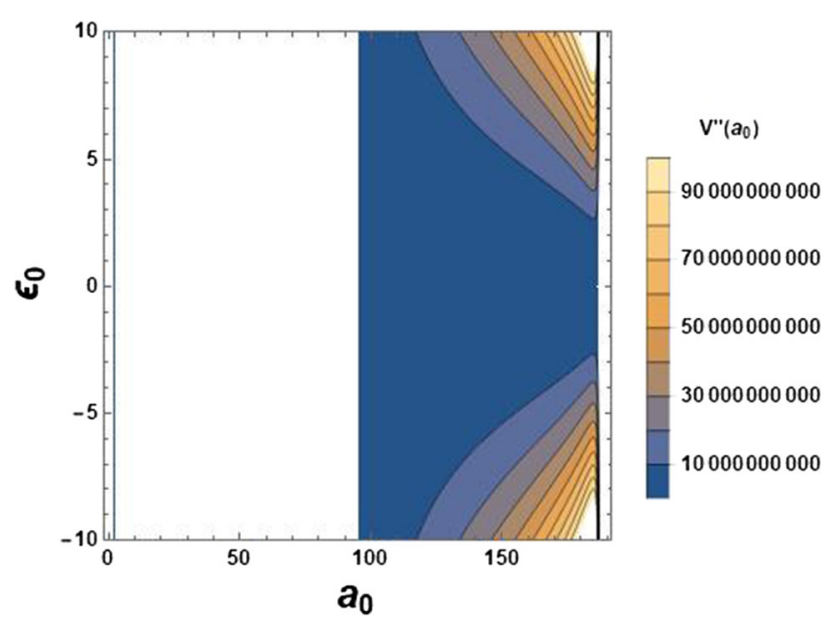

Fig. 3 The stable region of the Chaplygin gas model $p(\sigma)=-\epsilon_{0}\left(\frac{1}{\sigma}-\right.$ $\left.\frac{1}{\sigma_{0}}\right)$. The result shows that $\epsilon_{0}$ can have both negative values and positive ones in the throat with radius $a_{0}$

\subsection{Chaplygin gas model}

We next consider the Chaplygin gas model for the exotic matter. The pressure is already given in Ref. [56]:

$p(\sigma)=-\epsilon_{0}\left(\frac{1}{\sigma}-\frac{1}{\sigma_{0}}\right)$.

It is trivial to show that

$\frac{\mathrm{d} p}{\mathrm{~d} \sigma}=\frac{\epsilon_{0}}{\sigma_{0}^{2}}$,

where $\sigma_{0}$ is a constant. After substituting the above results into the stability condition (57), we find in this case

$$
\begin{aligned}
0< & \frac{1}{2} f^{\prime \prime}\left(a_{0}\right)+\frac{1}{4 a \sigma^{3} \sigma_{0}^{2}}\left(1 6 \pi \gamma \epsilon _ { 0 } \sigma _ { 0 } \left(\epsilon_{0}\left(\sigma-\sigma_{0}\right)\right.\right. \\
& \left.+\sigma^{2} \sigma_{0}\right)-a\left(\Lambda^{2} \sigma^{3} \sigma_{0}^{2}\right. \\
& +64 \pi^{2} \epsilon_{0} \sigma^{2}\left(\epsilon_{0}\left(\sigma-\sigma_{0}\right)+\sigma \sigma_{0}^{2}\right) \\
& \left.\left.+8 \pi \epsilon_{0} \Lambda \sigma_{0}\left(\epsilon_{0}\left(\sigma-\sigma_{0}\right)+\sigma^{2}\left(3 \sigma_{0}-2 \sigma\right)\right)\right)\right) .
\end{aligned}
$$

Here we plot the stability contour in terms of $\epsilon_{0}$ and $a_{0}$ for this model. The result is illustrated in Fig. 3. The stable region for this case is represented in Fig. 3. We notice that in order to satisfy the stability condition (57) $\epsilon_{0}$ can have both negative values and positive ones in the throat with radius $a_{0}$.

\subsection{Generalized Chaplygin gas model}

In addition, the Chaplygin gas model given in the previous subsection can be generalized where the relation between $p(\sigma)$ and $\sigma$ takes the form [56]

$p(\sigma)=-\left(\frac{\sigma_{0}}{\sigma}\right)^{\epsilon_{0}}$

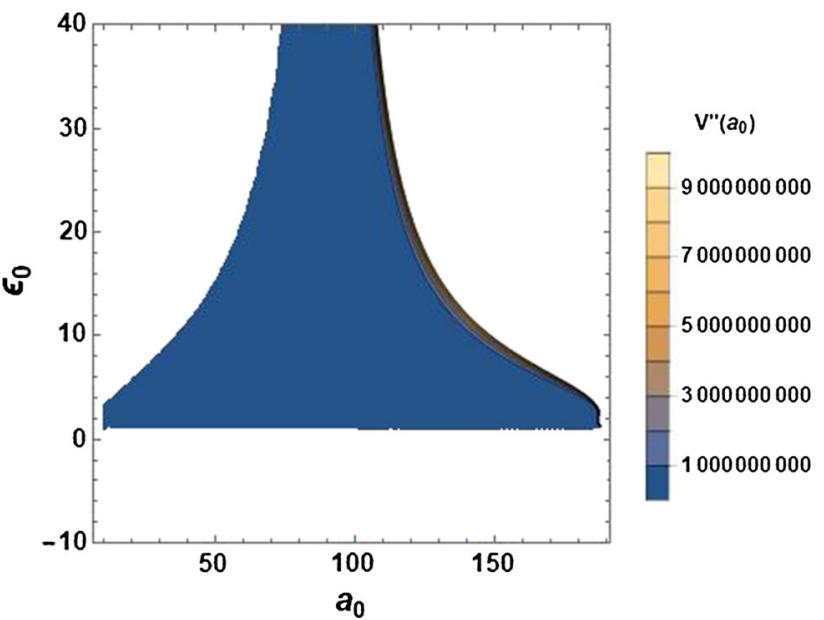

Fig. 4 The stable region of the generalized Chaplygin gas model $p(\sigma)=\left(\frac{\sigma_{0}}{\sigma}\right)^{\epsilon_{0}}$. The result shows that $\epsilon_{0}$ has positive values in the throat with $a_{0}$

and

$\frac{\mathrm{d} p}{\mathrm{~d} \sigma}=\epsilon_{0} \frac{\sigma_{0}^{\epsilon_{0}}}{\sigma^{\epsilon_{0}+1}}$.

After substituting the above results into the stability condition (57), we find in this case

$$
\begin{aligned}
0< & \frac{1}{2} f^{\prime \prime}\left(a_{0}\right)+\frac{1}{4 a} \sigma^{-1-\epsilon_{0}}\left(-64 a \pi^{2}\left(\frac{\sigma_{0}^{2 \epsilon_{0}}}{\sigma^{\epsilon_{0}-1}}\right)\right. \\
& +8 \pi\left(\frac{\sigma_{0}}{\sigma}\right)^{\epsilon_{0}}\left(-2 a \Lambda \sigma^{1+\epsilon_{0}}+\epsilon_{0}((-2 \gamma+a \Lambda)\right. \\
& \left.\left.+8 a \pi \sigma) \sigma_{0}^{\epsilon_{0}}\right)+\sigma\left(-8 \pi \epsilon_{0}((-2 \gamma+a \Lambda)+8 a \pi \sigma) \sigma_{0}^{\epsilon_{0}}\right)\right) \\
& -\frac{1}{4} \Lambda^{2} .
\end{aligned}
$$

Here we display the stability contour in terms of $\epsilon_{0}$ and $a_{0}$ illustrated in Fig. 4. The stable region for this case is represented in Fig. 4 for the generalized Chaplygin gas model. We find that in order to satisfy the stability condition (57) $\epsilon_{0}$ has positive values in the throat with radius $a_{0}$.

\subsection{Logarithm model}

We provide the last example in which the pressure $p(\sigma)$ and the energy density $\sigma$ are related via [56]

$p(\sigma)=\epsilon_{0} \log \left(\frac{\sigma}{\sigma_{0}}\right)$

and

$\frac{\mathrm{d} p}{\mathrm{~d} \sigma}=\frac{\epsilon_{0}}{\sigma}$. 


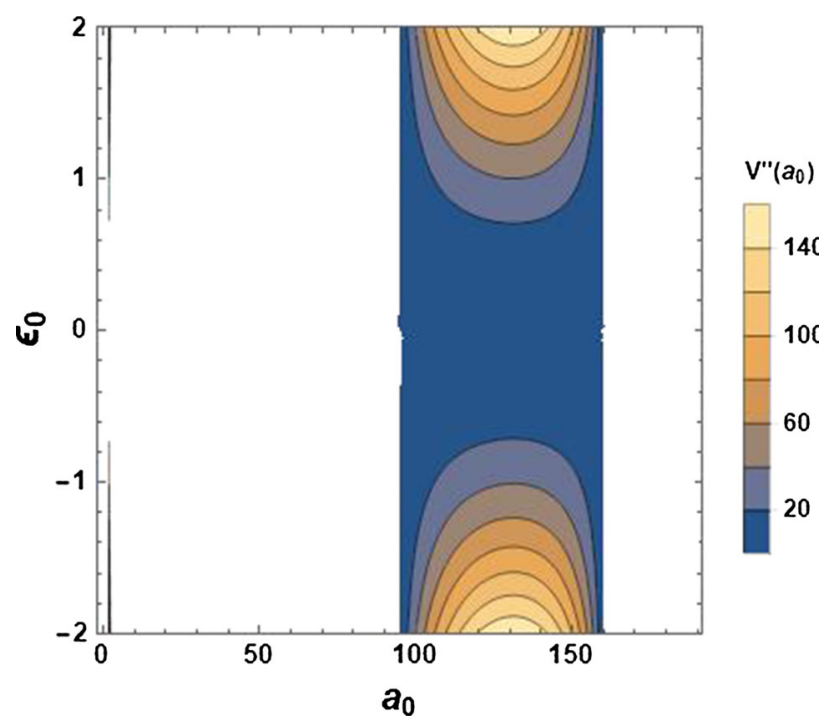

Fig. 5 The stable region of the linear model $p(\sigma)=\epsilon_{0} \log \left(\frac{\sigma}{\sigma_{0}}\right)$. The result shows that $\epsilon_{0}$ can have both negative values and positive ones in the throat with radius $a_{0}$

After substituting the above results into the stability condition (57), we find in this particular case

$$
\begin{gathered}
0<\frac{1}{2} f^{\prime \prime}\left(a_{0}\right)+\frac{4 \pi \gamma \epsilon_{0}\left(\sigma+\epsilon_{0} \log \left(\frac{\sigma}{\sigma_{0}}\right)\right)}{\sigma a} \\
-\frac{\Lambda^{2}+8 \pi \Lambda\left(\epsilon_{0}+\epsilon_{0}\left(\frac{\epsilon_{0}}{\sigma}-2\right) \log \left(\frac{\sigma}{\sigma_{0}}\right)\right)}{4 a} \\
-\frac{64 \pi^{2} \epsilon_{0}\left(\sigma+\epsilon_{0} \log \left(\frac{\sigma}{\sigma_{0}}\right)\left(1+\log \left(\frac{\sigma}{\sigma_{0}}\right)\right)\right)}{4 a} .
\end{gathered}
$$

Here we display the stability contour in terms of $\epsilon_{0}$ and $a_{0}$ illustrated in Fig. 5. The stable region for this case is represented in Fig. 5 for the logarithm model. We observe that in order to satisfy the stability condition (57) $\epsilon_{0}$ can have both negative values and positive ones in the throat with radius $a_{o}$.

\section{Energy conditions}

In this section, we shall analyze the energy conditions for the thin-shell wormholes in the dRGT massive gravity. We check the null, weak, and strong conditions at the wormhole throat for all existing models present in the previous section.

I. Null energy condition is expressed in terms of energy density and pressure as follows:

$$
\rho_{\text {eff. }}+P_{\text {eff. }} \geq 0
$$

which yields

$$
\begin{aligned}
\rho_{\text {eff. }}+P_{\text {eff. }}= & \sigma-\frac{1}{8 \pi G}\left(\frac{2 \gamma}{a}-\Lambda\right) \\
& +p+\frac{1}{8 \pi G}\left(\frac{\gamma}{a}-\Lambda\right) \\
= & \sigma+p-\frac{1}{8 \pi G} \frac{\gamma}{a} \geq 0 .
\end{aligned}
$$

II. Weak energy condition is given by

$\rho_{\text {eff. }} \geq 0, \quad \rho_{\text {eff. }}+P_{\text {eff. }} \geq 0$

which gives the following result for the thin-shell wormholes in the dRGT massive gravity:

$\rho_{\text {eff. }}=\sigma-\frac{1}{8 \pi G}\left(\frac{2 \gamma}{a}-\Lambda\right) \geq 0$,

III. Strong energy condition is governed by

$\rho_{\text {eff. }}+3 P_{\text {eff. }} \geq 0, \quad \rho_{\text {eff. }}+P_{\text {eff. }} \geq 0$,

which gives the following result for the thin-shell wormholes in the dRGT massive gravity:

$$
\begin{aligned}
\rho_{\text {eff. }}+3 P_{\text {eff. }}= & \sigma-\frac{1}{8 \pi G}\left(\frac{2 \gamma}{a}-\Lambda\right) \\
& +3 p+\frac{3}{8 \pi G}\left(\frac{\gamma}{a}-\Lambda\right) \\
= & \sigma+3 p+\frac{1}{8 \pi G}\left(\frac{\gamma}{a}-2 \Lambda\right) \geq 0 .
\end{aligned}
$$

\subsection{Linear model}

When substituting the pressure and the energy density of this model into Eqs. (75), (77) and (79), we find

$$
\begin{aligned}
\rho_{\text {eff. }}+P_{\text {eff. }} & =\frac{\left(\left(1+2 \epsilon_{0}\right) \gamma-a\left(1+\epsilon_{0}\right) \Lambda\right)-2\left(1+\epsilon_{0}\right) \sqrt{f(a)}}{8 \pi G a} \\
& \geq 0, \\
\rho_{\text {eff. }} & =-\frac{\sqrt{f(a)}}{4 \pi G a} \geq 0, \\
\rho_{\text {eff. }}+3 P_{\text {eff. }} & =\frac{3\left(\left(1+2 \epsilon_{0}\right) \gamma-a\left(1+\epsilon_{0}\right) \Lambda\right)-2\left(1+\epsilon_{0}\right) \sqrt{f(a)}}{8 \pi G a} \\
& \geq 0 .
\end{aligned}
$$

In order to analyze the energy conditions, we will choose the values of $\epsilon_{0}$ in the stable regions shown in Fig. 2 and then verify the energy conditions. Figure 6 shows the variation of $\rho_{\text {eff. }}+P_{\text {eff. }}, \rho_{\text {eff. and }} \rho_{\text {eff. }}+3 P_{\text {eff. as a function of } a \text { in }}$ the linear model $p(\sigma)=\epsilon_{0} \sigma$. We observe that all energy conditions are violated in this model. 

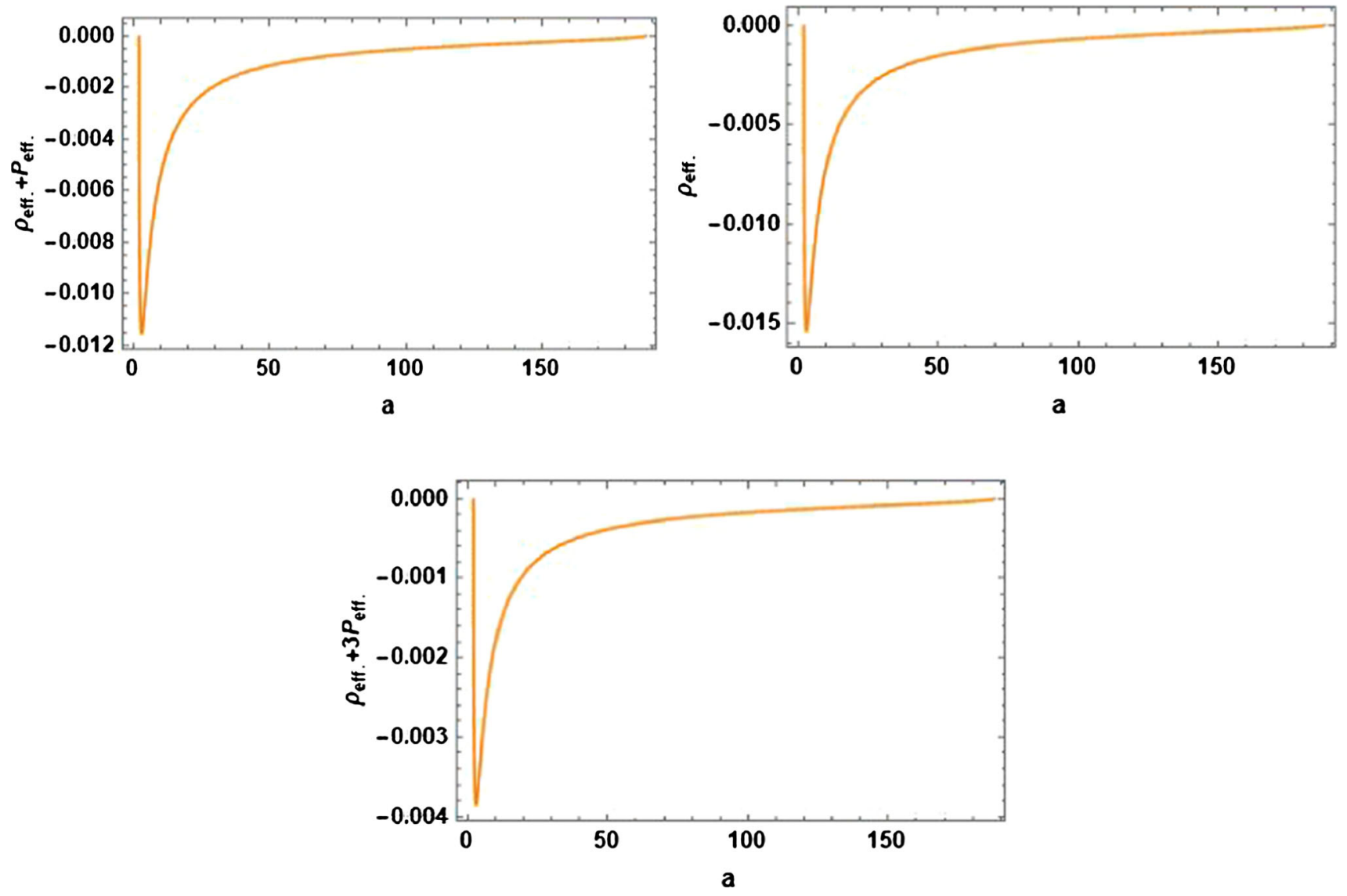

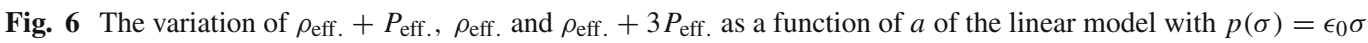

\subsection{Chaplygin gas model}

We substitute the pressure and the energy density of this model into Eqs. (75), (77) and (79) and then we obtain

$$
\begin{aligned}
& \rho_{\text {eff. }}+P_{\text {eff. }}=\frac{1}{8}\left(\frac{(\gamma-a \Lambda)}{\pi G a}\right. \\
&- \frac{8 \epsilon_{0}\left((-2 \gamma+a \Lambda)+8 a G \pi \sigma_{0}+2 \sqrt{f(a)}\right)}{\sigma_{0}((2 \gamma-a \Lambda)-2 \sqrt{f(a)})} \\
&-\left.\frac{2 \sqrt{f(a)}}{a G \pi}\right) \geq 0, \\
& \rho_{\text {eff. }}=-\frac{\sqrt{f(a)}}{4 \pi G a} \geq 0, \\
& \rho_{\text {eff. }}+3 P_{\text {eff. }}=\frac{1}{8}\left(\frac{3(\gamma-a \Lambda)}{\pi G a}\right. \\
&-\frac{24 \epsilon_{0}\left((-2 \gamma+a \Lambda)+8 a G \pi \sigma_{0}+2 \sqrt{f(a)}\right)}{\sigma_{0}((2 \gamma-a \Lambda)-2 \sqrt{f(a)})} \\
&\left.-\frac{2 \sqrt{f(a)}}{a G \pi}\right) \geq 0 .
\end{aligned}
$$

In order to quantify the energy conditions, we will choose the values of $\epsilon_{0}$ in the stable regions shown in Fig. 3 and then examine the energy conditions. Figure 7 shows the variation of $\rho_{\text {eff. }}+P_{\text {eff. }}, \rho_{\text {eff. and }} \rho_{\text {eff. }}+3 P_{\text {eff. }}$ as a function of $a$ in the linear model $p(\sigma)=-\epsilon_{0}\left(\frac{1}{\sigma}-\frac{1}{\sigma_{0}}\right)$. We observe that all energy conditions are violated for $a<100$ in this model.

\subsection{Generalized Chaplygin gas model}

When substituting the pressure and the energy density of this model into Eqs. (75), (77) and (79), we find

$$
\begin{aligned}
& \rho_{\text {eff. }}+P_{\text {eff. }} \\
&= \frac{1}{8 \pi G a}((\gamma-a \Lambda)-2 \sqrt{f(a)} \\
&\left.-G a(8 \pi)^{1+\epsilon_{0}}\left(-\frac{G a \sigma_{0}}{(-2 \gamma+a \Lambda)+2 \sqrt{f(a)}}\right)^{\epsilon_{0}}\right) \\
& \geq 0, \\
& \rho_{\text {eff. }}=-\frac{\sqrt{f(a)}}{4 \pi G a} \geq 0, \\
& \rho_{\text {eff. }}+3 P_{\text {eff. }} \\
&=\frac{1}{8 \pi G a}(3(\gamma-3 a \Lambda)-2 \sqrt{f(a)} \\
&\left.\quad-3 G a(8 \pi)^{1+\epsilon_{0}}\left(-\frac{G a \sigma_{0}}{(-2 \gamma+a \Lambda)+2 \sqrt{f(a)}}\right)^{\epsilon_{0}}\right) \\
& \geq 0 .
\end{aligned}
$$



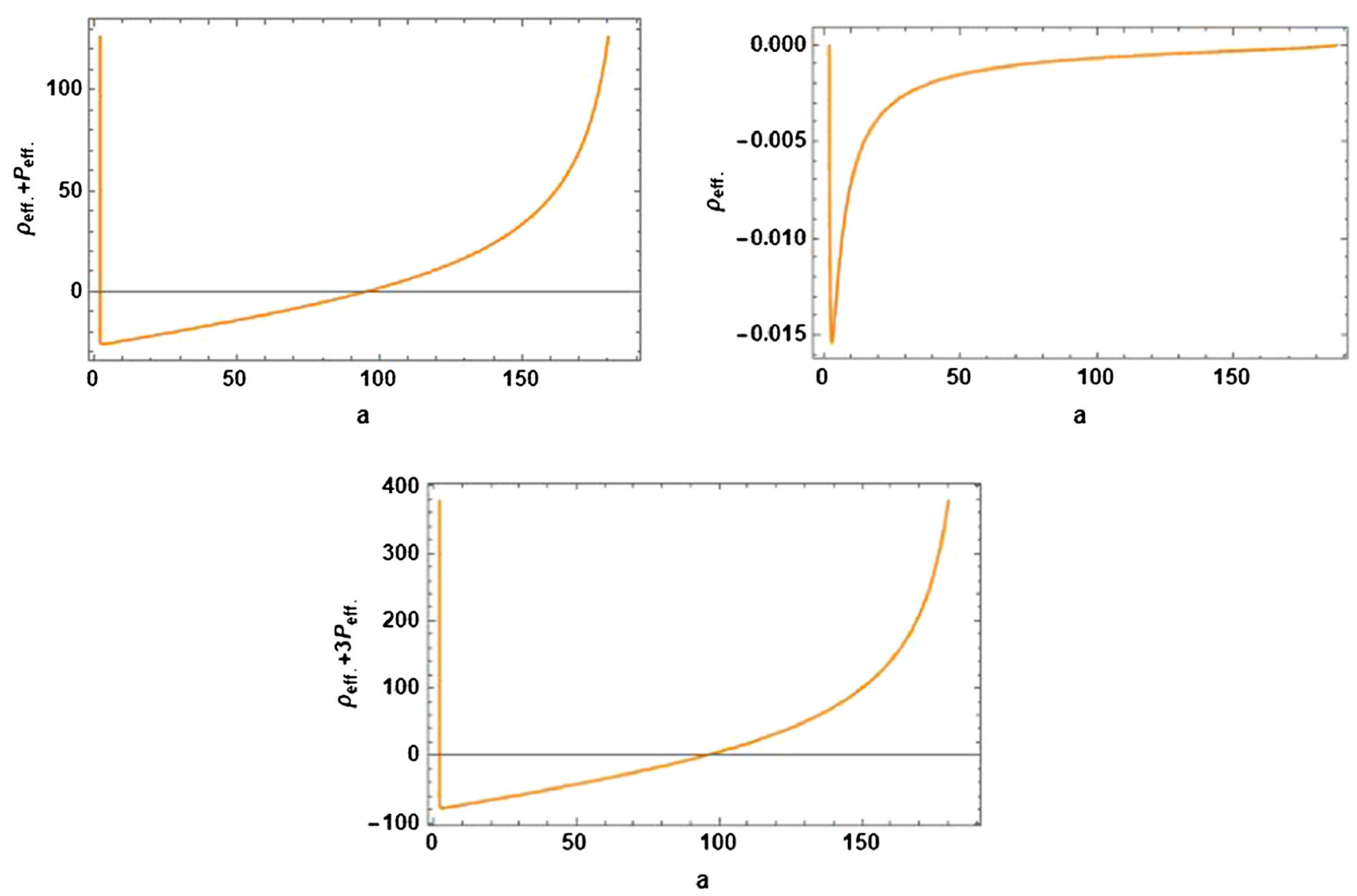

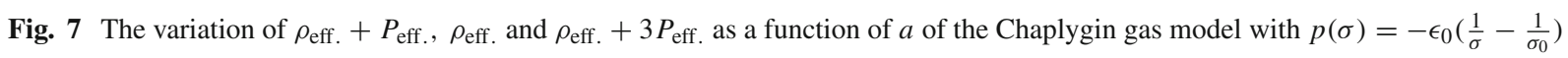

We here quantify the energy conditions by choosing the values of $\epsilon_{0}$ in the stable regions shown in Fig. 4 and then examine the energy conditions. Figure 8 shows the variation of $\rho_{\text {eff. }}+P_{\text {eff., }} \rho_{\text {eff. and }} \rho_{\text {eff. }}+3 P_{\text {eff. as a function of } a \text { in }}$ the generalized Chaplygin gas model $p(\sigma)=\left(\frac{\sigma_{0}}{\sigma}\right)^{\epsilon_{0}}$. We observe that all energy conditions are violated for positive values of $\epsilon_{0}$.

\subsection{Logarithm model}

We substitute the pressure and the energy density of this model into Eqs. (75), (77) and (79) and then we obtain

$$
\begin{aligned}
\rho_{\text {eff. }}+P_{\text {eff. }}= & \frac{(\gamma-a \Lambda)-2 \sqrt{f(a)}}{8 \pi G a} \\
& +\epsilon_{0} \log \left(\frac{(2 \gamma-a \Lambda)-2 \sqrt{f(a)}}{8 \pi G a \sigma_{0}}\right) \\
\geq & 0, \\
\rho_{\text {eff. }}= & -\frac{\sqrt{f(a)}}{4 \pi G a} \geq 0, \\
\rho_{\text {eff. }}+3 P_{\text {eff. }}= & \frac{3(\gamma-a \Lambda)-2 \sqrt{f(a)}}{8 \pi G a}
\end{aligned}
$$

$$
\begin{aligned}
& +3 \epsilon_{0} \log \left(\frac{(2 \gamma-a \Lambda)-2 \sqrt{f(a)}}{8 \pi G a \sigma_{0}}\right) \\
\geq & 0 .
\end{aligned}
$$

We here quantify the energy conditions by choosing the values of $\epsilon_{0}$ in the stable regions shown in Fig. 5 and then examine the energy conditions. Figure 9 shows the variation of $\rho_{\text {eff. }}+P_{\text {eff. }}, \rho_{\text {eff. and }} \rho_{\text {eff. }}+3 P_{\text {eff. }}$ as a function of $a$ in the logarithm model $p(\sigma)=\epsilon_{0} \log \left(\frac{\sigma}{\sigma_{0}}\right)$. We observe that all energy conditions are violated for positive values of $\epsilon_{0}$ with $a>100$.

\section{Embedding diagram}

In this section, we construct the wormhole geometry via the embedding diagrams to represent a thin-shell wormhole in the dRGT massive gravity and extract some useful information by considering an equatorial slice, $\theta=\pi / 2$ and a fixed moment of time, $t=$ const . . Therefore the metric reduces to

$\mathrm{d} s^{2}=\frac{\mathrm{d} r^{2}}{f(r)}+r^{2} \mathrm{~d} \phi^{2}$ 

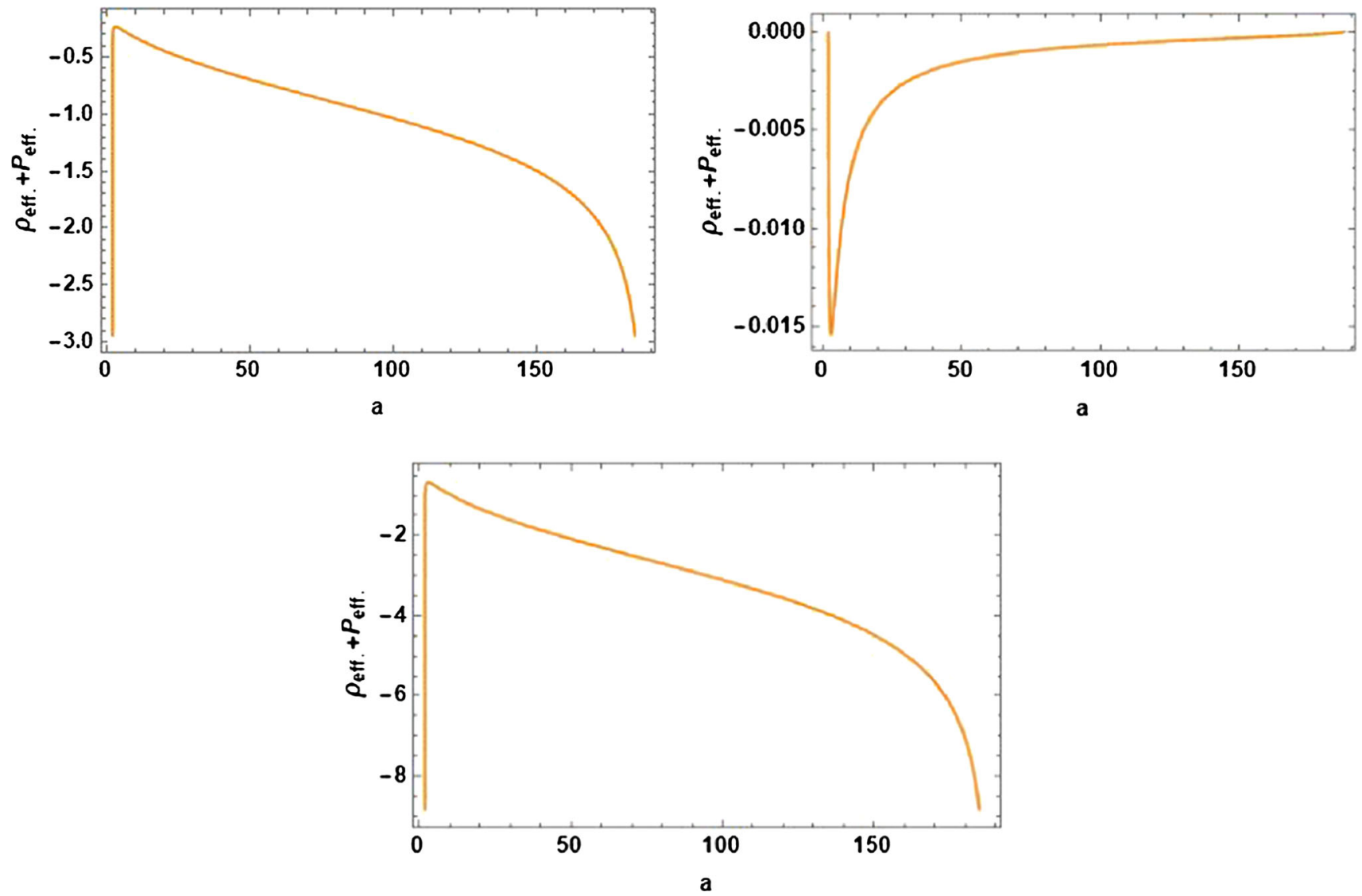

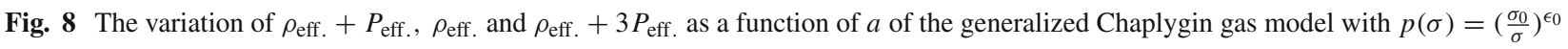

where

$f(r)=1-\frac{2 M}{r}-\frac{\Lambda r^{2}}{3}+\gamma r+\zeta$

Next we embed the metric from Eq. (93) into 3-dimensional Euclidean space to visualize this slice and hence the spacetime can be written in cylindrical coordinates as

$$
\begin{aligned}
\mathrm{d} s^{2} & =\mathrm{d} z^{2}+\mathrm{d} r^{2}+r^{2} \mathrm{~d} \phi^{2} \\
& =\left(1+\left(\frac{\mathrm{d} z}{\mathrm{~d} r}\right)^{2}\right) \mathrm{d} r^{2}+r^{2} \mathrm{~d} \phi^{2}
\end{aligned}
$$

Comparing Eq. (92) with Eq. (94) generates the expression for the embedding surface, which is given by

$\frac{\mathrm{d} z}{\mathrm{~d} r}= \pm \sqrt{\frac{1-f(r)}{f(r)}}$.

where $f(r)$ is given in Eq. (93). However, the integration of the above expression cannot be solved analytically. Performing a numerical technique allows us to illustrate the wormhole shape as in Fig. 10.

\section{Discussions and conclusions}

In this work, we have studied the thin-shell wormholes in dRGT massive gravity. In order to construct the thin-shell wormhole, two bulks of the spacetime geometry are glued together via the cut-and-paste procedure [52]. Moreover, the junction conditions of dRGT spacetime are also derived in this work. The massive graviton correction term of the dRGT theory, $m_{g}^{2} \mathcal{U}\left(g, \phi^{2}\right)$, in the Einstein equation is represented in terms of effective anisotropic pressure fluid. However, if there is only this correction term, without invoking exotic fluids, we have found that the thin-shell wormholes cannot be stabilized. We have also quantified the dynamics of the spherical thin-shell wormholes in the dRGT theory. We then examined the stability conditions of the wormholes by introducing four existing models of the exotic fluids at the throat. In addition, we analyzed the energy conditions for the thinshell wormholes in the dRGT massive gravity by checking the null, weak, and strong conditions at the wormhole throat.

We have quantified the energy conditions of the four models by choosing the values of $\epsilon_{0}$ in the stable regions shown in Sect. 4 . We have considered the variation of $\rho_{\text {eff. }}+P_{\text {eff. }}, \rho_{\text {eff }}$.

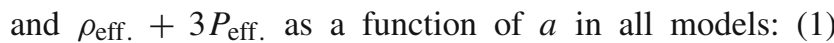



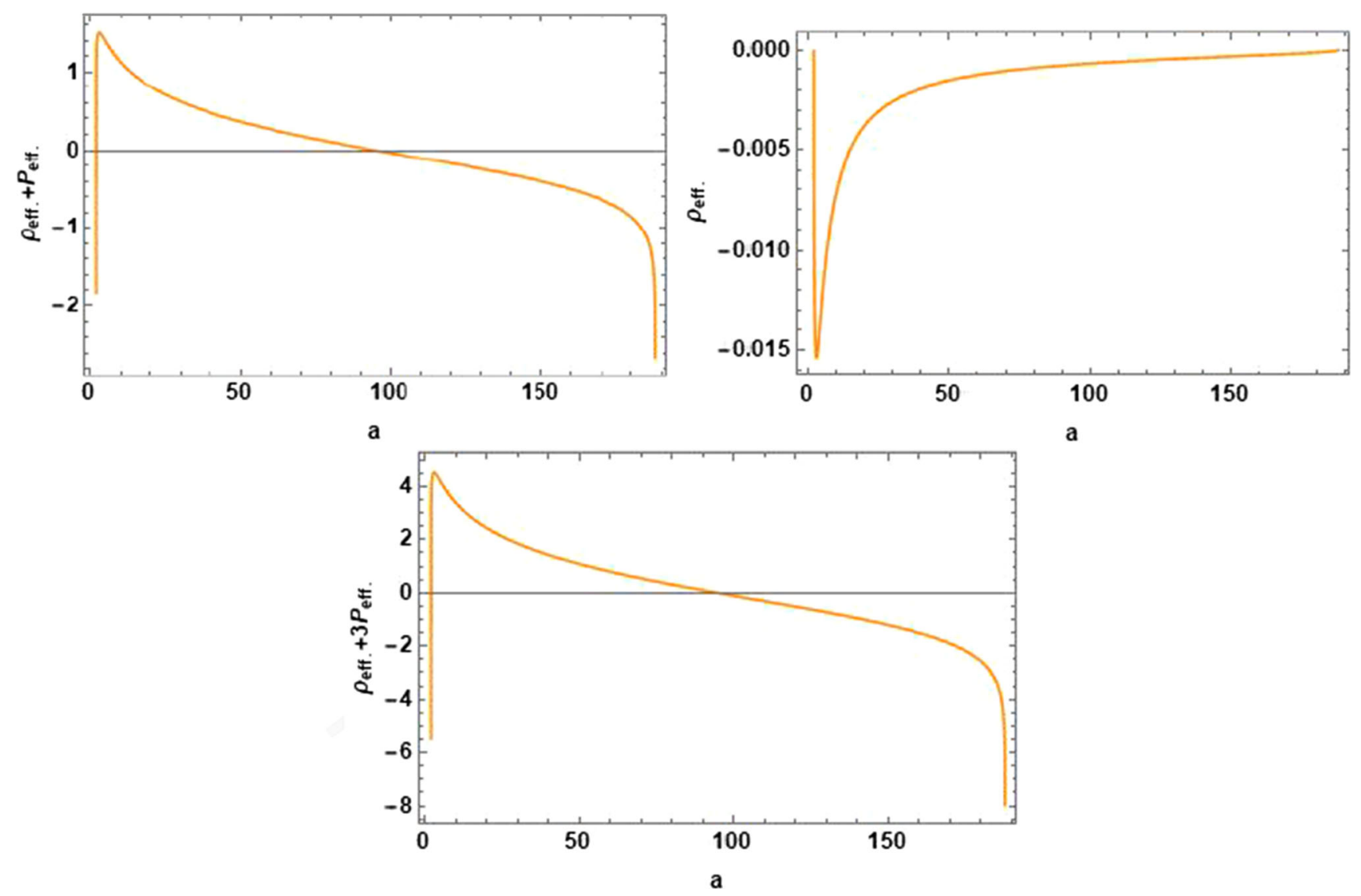

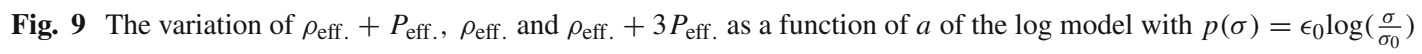

the linear model $p(\sigma)=\left(\frac{\sigma_{0}}{\sigma}\right)^{\epsilon_{0}}$, (2) the Chaplygin gas model $p(\sigma)=-\epsilon_{0}\left(\frac{1}{\sigma}-\frac{1}{\sigma_{0}}\right)$, (3) the generalized Chaplygin gas model $p(\sigma)=\left(\frac{\sigma_{0}}{\sigma}\right)^{\epsilon_{0}}$ and (4) the logarithm model $p(\sigma)=\epsilon_{0} \log \left(\frac{\sigma}{\sigma_{0}}\right)$. Choosing the values of $\epsilon_{0}$ in the stable regions, we have observed that in general the classical energy conditions are violated by introducing all existing models of the exotic fluids. Moreover, we have quantified the wormhole geometry by using the embedding diagram to represent a thin-shell wormhole in the dRGT massive gravity.

However, there are some limitations in the present workfor example, the construction of the shadow cast by the thinshell wormhole in the dRGT massive gravity is worth investigating. Regarding this, we can evaluate the test particle geodesics and determine the trajectories of photons around the wormhole. This can be straightforwardly done by following the work of Refs. [16,21,62]. Additionally, we can elaborate our work by studying the gravitational lensing effect in the spacetime of the wormhole metric (10). This allows us to determine the deflection angle of the photon due to the presence of the wormhole in the dRGT massive gravity. It is worth mentioning that gravitational lensing and particle motions around non-asymptotically flat black hole spacetime in dRGT massive gravity have been addressed in Ref. [63].

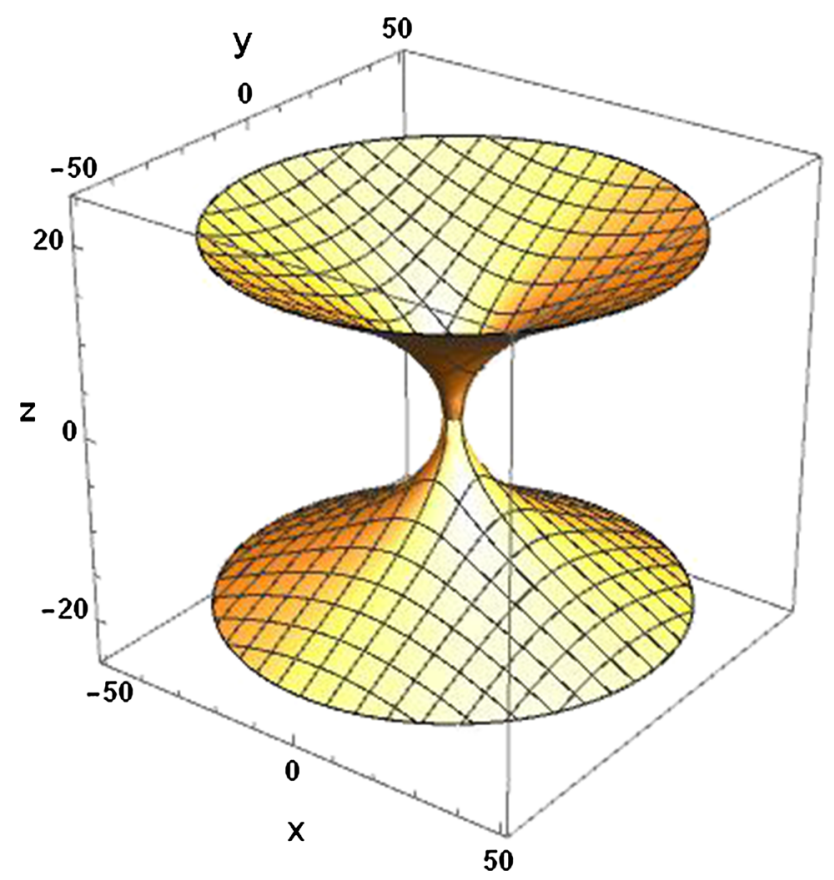

Fig. 10 The wormhole shape obtained via the embedding diagrams in 3-dimensional asymptotic flat spacetime. We have used $M=1, \Lambda=$ $0.0001, \gamma=0.001$ and set $\zeta=0$ 
Acknowledgements TT thanks the Science Achievement Scholarship of Thailand (SAST) for financial support during his PhD study. $\mathrm{AC}$ is supported by the CUniverse research promotion project of Chulalongkorn University under the grant reference CUAASC. DS is supported by Thailand Research Fund (TRF) under a contract No.TRG6180014. PC thanks Kimet Jusufi for initiating research collaboration on the wormhole issues.

Data Availability Statement This manuscript has no associated data or the data will not be deposited. [Authors' comment: We do not have any data related to this manuscript. It is a theoretical work only and no data is involved.]

Open Access This article is licensed under a Creative Commons Attribution 4.0 International License, which permits use, sharing, adaptation, distribution and reproduction in any medium or format, as long as you give appropriate credit to the original author(s) and the source, provide a link to the Creative Commons licence, and indicate if changes were made. The images or other third party material in this article are included in the article's Creative Commons licence, unless indicated otherwise in a credit line to the material. If material is not included in the article's Creative Commons licence and your intended use is not permitted by statutory regulation or exceeds the permitted use, you will need to obtain permission directly from the copyright holder. To view a copy of this licence, visit http://creativecomm ons.org/licenses/by/4.0/.

Funded by SCOAP ${ }^{3}$.

\section{References}

1. K. Schwarzschild, Sitzungsber. Preuss. Akad. Wiss. Berl. (Math. Phys.) 1916, 189 (1916)

2. B.P. Abbott et al. (LIGO Scientific and Virgo Collaborations), Phys. Rev. Lett. 116(6), 061102 (2016)

3. L. Flamm, Physikalishe Zeitschrift 17, 448 (1916). Note: This article is unavailable online. The editorial note on the Ludwig Flamm's work is given online: G. W. Gibbons, Gen. Relativ. Gravit. 47, 71 (2015)

4. A. Einstein, N. Rosen, Phys. Rev. 48, 73 (1935)

5. C.W. Misner, J.A. Wheeler, Ann. Phys. 2, 525 (1957)

6. H.G. Ellis, J. Math. Phys. 14, 104 (1973)

7. K.A. Bronnikov, Acta Phys. Pol. B 4, 251 (1973)

8. M.S. Morris, K.S. Thorne, Am. J. Phys. 56, 395 (1988)

9. M.S. Morris, K.S. Thorne, U. Yurtsever, Phys. Rev. Lett. 61, 1446 (1988)

10. T. Harko, F.S.N. Lobo, M.K. Mak, S.V. Sushkov, Mod. Phys. Lett. A 30(35), 1550190 (2015)

11. F.S.N. Lobo, M.A. Oliveira, Phys. Rev. D 80, 104012 (2009)

12. C.G. Boehmer, T. Harko, F.S.N. Lobo, Phys. Rev. D 85, 044033 (2012)

13. M. Kord Zangeneh, F.S.N. Lobo, M.H. Dehghani, Phys. Rev. D 92(12), 124049 (2015)

14. G. Clement, Gen. Relativ. Gravit. 16, 131 (1984)

15. K.A. Bronnikov, M.V. Skvortsova, A.A. Starobinsky, Gravit. Cosmol. 16, 216 (2010)

16. K. Jusufi, N. Sarkar, F. Rahaman, A. Banerjee, S. Hansraj, Eur. Phys. J. C 78(4), 349 (2018)

17. K. Jusufi, Eur. Phys. J. C 76(11), 608 (2016)

18. A. Övgün, K. Jusufi, İ. Sakall, Phys. Rev. D 99(2), 024042 (2019)

19. R. Shaikh, Phys. Rev. D 98(6), 064033 (2018)

20. R. Shaikh, S. Kar, Phys. Rev. D 94(2), 024011 (2016)

21. A. Övgün, G. Gyulchev, K. Jusufi, Ann. Phys. 406, 152 (2019)

22. N. Montelongo Garcia, F.S.N. Lobo, Class. Quantum Gravity 28, 085018 (2011)
23. F. Rahaman, N. Paul, A. Banerjee, S.S. De, S. Ray, A.A. Usmani, Eur. Phys. J. C 76(5), 246 (2016)

24. F. Rahaman, I. Karar, S. Karmakar, S. Ray, Phys. Lett. B 746, 73 (2015)

25. M. Jamil, F. Rahaman, R. Myrzakulov, P.K.F. Kuhfittig, N. Ahmed, U.F. Mondal, J. Korean Phys. Soc. 65(6), 917 (2014)

26. F. Rahaman, S. Islam, P.K.F. Kuhfittig, S. Ray, Phys. Rev. D 86, $106010(2012)$

27. S. Bahamonde, U. Camci, S. Capozziello, M. Jamil, Phys. Rev. D 94(8), $084042(2016)$

28. S. Bahamonde, M. Jamil, P. Pavlovic, M. Sossich, Phys. Rev. D 94(4), 044041 (2016)

29. M. Jamil, D. Momeni, R. Myrzakulov, Eur. Phys. J. C 73, 2267 (2013)

30. M. Jamil, U. Farooq, M.A. Rashid, Eur. Phys. J. C 59, 907 (2009)

31. F.S.N. Lobo, Phys. Rev. D 71, 084011 (2005)

32. P. Kanti, B. Kleihaus, J. Kunz, Phys. Rev. Lett. 107, 271101 (2011)

33. R. Garattini, Eur. Phys. J. C 79, 951 (2019)

34. M. Halilsoy, A. Ovgun, S.H. Mazharimousavi, Eur. Phys. J. C 74, $2796(2014)$

35. A. Ovgun, Eur. Phys. J. Plus 131(11), 389 (2016)

36. M.G. Richarte, I.G. Salako, J.P. Morais Graça, H. Moradpour, A. Övgün, Phys. Rev. D 96(8), 084022 (2017)

37. M. Fierz, W. Pauli, Proc. R. Soc. Lond. A 173, 211 (1939)

38. V.I. Zakharov, JETP Lett. 12, 312 (1970). [Pisma Zh. Eksp. Teor. Fiz. 12, 447 (1970)]

39. H. van Dam, M.J.G. Veltman, Nucl. Phys. B 22, 397 (1970)

40. A.I. Vainshtein, Phys. Lett. 39B, 393 (1972)

41. K. Hinterbichler, Rev. Mod. Phys. 84, 671 (2012)

42. C. de Rham, G. Gabadadze, A.J. Tolley, Phys. Rev. Lett. 106, $231101(2011)$

43. S.G. Ghosh, L. Tannukij, P. Wongjun, Eur. Phys. J. C 76(3), 119 (2016)

44. M. Chabab, H. El Moumni, S. Iraoui, K. Masmar, Eur. Phys. J. C 79(4), 342 (2019)

45. P. Boonserm, T. Ngampitipan, P. Wongjun, Eur. Phys. J. C 78(6), 492 (2018)

46. A.G. Riess et al. (Supernova Search Team), Astron. J. 116, 1009 (1998)

47. S. Perlmutter et al. (Supernova Cosmology Project Collaboration), Astrophys. J. 517, 565 (1999)

48. C. de Rham, G. Gabadadze, Phys. Rev. D 82, 044020 (2010)

49. P. Burikham, T. Harko, M.J. Lake, Phys. Rev. D 94(6), 064070 (2016)

50. P. Kareeso, P. Burikham, T. Harko, Eur. Phys. J. C 78(11), 941 (2018)

51. S. Panpanich, P. Burikham, Phys. Rev. D 98(6), 064008 (2018)

52. M. Visser, Phys. Rev. D 39, 3182 (1989)

53. M. Visser, Lorentzian Wormholes: From Einstein to Hawking (AIP, Woodbury, 1995), p. 412

54. L. Avilés, H. Maeda, C. Martinez, Class. Quantum Gravity 37, 075022 (2020)

55. E. Poisson, A Relativist's Toolkit: The Mathematics of Black-Hole Mechanics (Cambridge University Press, 2004)

56. A. Övgün. arXiv: 1610.08118 [gr-qc]

57. T. Kokubu, Stability of wormholes with singular hypersurfaces in Einstein and Gauss-Bonnet theories of gravity, Ph.D. thesis

58. S.W. Kim, Phys. Lett. B 780, 174 (2018)

59. A. Padilla, V. Sivanesan, JHEP 1208, 122 (2012)

60. M. Tanabashi et al. (Particle Data Group), Phys. Rev. D 98(3), 030001 (2018)

61. M. Ishak, K. Lake, Phys. Rev. D 65, 044011 (2002)

62. M. Amir, K. Jusufi, A. Banerjee, S. Hansraj, Class. Quantum Gravity 36(21), 215007 (2019)

63. S. Panpanich, S. Ponglertsakul, L. Tannukij, Phys. Rev. D 100(4), 044031 (2019) 